\title{
Localized temporal variation of Earth's inner-core boundary from high-quality waveform doublets
}

\author{
Danhua Xin $\cdot$ Xiaodong Song $\cdot$ Tao Wang
}

Received: 2 April 2015/ Accepted: 27 May 2015/Published online: 16 June 2015

(C) The Author(s) 2015. This article is published with open access at Springerlink.com

\begin{abstract}
The topography of the inner core is crucial to understand its growth process and interaction with the geodynamo. With the accuracy of teleseismic waveform doublets in determining the travel-time shifts between PKPcd and PKPdf inner-core phases, we examined the temporal change of the inner-core boundary sampled by new earthquake doublets that occurred in the Western Pacific and those previously found from the South Sandwich Islands. The receiver stations are those within the distance range of $128^{\circ}-142^{\circ}$ from the hypocenters of the waveform doublets. Our results suggest that temporal changes in PKPcd-PKPdf differential times are very subtle except some isolated regions under central America and Africa. The observations may indicate localized topography of the inner core and/or the inner-core boundary as transient slurry in isolated regions.
\end{abstract}

Keywords Waveform doublet · Inner-core boundary topography $\cdot$ Temporal change $\cdot$ PKP

\section{Introduction}

The Earth's inner core was gradually generated from the solidification of the liquid outer core (Jacobs 1953). The

D. Xin $\cdot$ X. Song $(\bowtie) \cdot T$. Wang

Institute of Geophysics and Geodynamics, School of Earth

Sciences and Engineering, Nanjing University, Nanjing, China

e-mail: xiao.d.song@gmail.com

\section{Song}

Department of Geology, University of Illinois at Urbana-

Champaign, Champaign, USA latent heat and light elements released at the inner-core boundary (ICB) during the solidification process provided the source of the outer core convection and affected the generation of the geodynamo (Glatzmaier and Roberts 1995). The growth of the inner core is geologically slow (Fearn et al. 1981; Loper and Roberts 1981; Stevenson et al. 1983; Yoshida et al. 1996) and geographically uniform because of the presumed extremely small variation in temperature in the outer core (Stevenson 1987). However, seismological studies have suggested structural and topographic complexities of the ICB. The existence of strong scattering from the inner core (Vidale et al. 2000; Koper et al. 2004) suggests heterogeneity at a scale length of tens of kilometers, which may be partly responsible for the attenuation of the waveforms passing through the inner core (Cormier et al. 1998). The variability of pre-critical PKPcd (or PKiKP) amplitudes has been used to infer the mosaic structure of the ICB (Krasnoshchekov et al. 2005), which includes patches of a thin partially liquid layer and patches of a sharp transition. The temporal changes of inner-core scattering waves were used to confirm (Vidale and Earle 2005) inner-core rotation (Song and Richards 1996). The bottom of the outer core has anomalous velocity gradients (Souriau and Poupinet 1991; Song and Helmberger 1995) and the topmost inner core shows hemispherical variation in seismic velocity (Niu and Wen 2001; Irving and Deuss 2011) or a transitional ICB (Jiang and Zhao 2012). In addition, evidence for significant topography of the ICB has been reported (Wen 2006; Cao et al. 2007; Dai and Song 2008; Dai et al. 2012). Imaging the structure of the inner-core boundary is important to understand the growth of the inner core and its interaction with the geodynamo of the fluid outer core. 
The accurate determination of the topography of an Earth's internal boundary is difficult because of the possible trade-off with the velocity of the media above it. Here we use waveform-doublet method to map the ICB topography. A waveform doublet is a pair of earthquakes occurring at essentially the same spatial position and received by the same station with high similarity in their waveforms (Poupinet et al. 1984), which make the exact detection of the ICB topography possible. This method has been used before in the detection of the inner-core rotation (Song and Richards 1996; Zhang et al. 2005), the topography of the ICB (Wen 2006; Cao et al. 2007; Song and Dai 2008) as well as the heterogeneity and motion of the Earth's liquid outer core (Dai and Song 2008). Waveform doublets can provide strong constraint on temporal changes by avoiding artifacts of event mislocation and contamination from mantle heterogeneities. In this study, we used this method to detect temporal change of the ICB using doublets from the Western Pacific (WP) area to increase global coverage of the ICB. Compared with previous study using doublets from South Sandwich Islands (SSI) (Song and Dai 2008), the new samples showed negligible temporal change of the ICB.

\section{Data and methods}

\subsection{Doublet search}

Our data were obtained from the IRIS DMC. We used vertical component of broadband stations, commonly sampled at $20 \mathrm{~Hz}$. We removed the original station response from the broadband waveform and convolved with the WWSSN (World-Wide Standardized Seismograph Network) short-period response. To enhance the SNR (signal-to-noise ratio), a causal second-order Butterworth band-pass filter from 0.6 to $3.0 \mathrm{~Hz}$ was applied to all the waveforms.

We first searched possible doublets for all the earthquakes that occurred in the WP area and recorded at station CHTO (Chiang Mai, Thailand) of the Global Seismic Network. The station was selected because of its generally high-quality data and the long operation history (it has operated as a broadband digital station since October, 1992). We used earthquakes from the catalog of the Preliminary Determination of Earthquakes (PDEs) of the U.S. Geological Survey. All the events that occurred from 1990s to December, 2013 with magnitudes of 5.3 and above were examined.
We used the cross-correlation (CC) method in determining the similarity of waveforms of each event pair. The waveform time window for the $\mathrm{CC}$ calculation is $5 \mathrm{~s}$ before and $35 \mathrm{~s}$ after predicted P-wave arrival time for the Preliminary Reference Earth Model (PREM) (Dziewonski and Anderson 1981). To improve the efficiency of the

Table 1 Detailed information of earthquakes used in Western Pacific area

\begin{tabular}{|c|c|c|c|c|c|}
\hline Date & Time & Lat. $\left(^{\circ}\right)$ & Long. $\left({ }^{\circ}\right)$ & Depth $(\mathrm{km})$ & $m_{\mathrm{b}}$ \\
\hline 1993-10-13 & $01: 39: 12.3$ & -6.426 & 154.934 & 102 & 5.5 \\
\hline 1994-08-05 & 03:39:05.3 & -3.177 & 101.466 & 68 & 5.4 \\
\hline $1997-01-24$ & $05: 17: 29.7$ & -5.614 & 153.836 & 70 & 5.6 \\
\hline 1998-08-17 & $12: 43: 39.8$ & -7.580 & 107.199 & 76 & 5.4 \\
\hline 2001-01-07 & $12: 55: 46.9$ & -8.703 & 108.893 & 33 & 5.4 \\
\hline $2004-12-27$ & $00: 32: 16.5$ & 5.476 & 94.467 & 33 & 5.8 \\
\hline 2004-12-31 & $14: 38: 46.6$ & 5.113 & 94.857 & 49 & 5.4 \\
\hline 2005-01-05 & $14: 34: 31.3$ & 5.588 & 94.759 & 30 & 5.3 \\
\hline 2005-01-06 & $00: 56: 29.9$ & 5.323 & 94.835 & 49 & 5.7 \\
\hline $2005-02-13$ & 01:22:09.3 & 5.079 & 94.794 & 48 & 5.3 \\
\hline $2005-03-13$ & $22: 12: 45.8$ & 5.486 & 94.595 & 52 & 5.5 \\
\hline $2005-05-21$ & $23: 01: 16.1$ & 5.281 & 94.797 & 55 & 5.6 \\
\hline 2005-06-17 & $02: 37: 39.3$ & 5.619 & 94.752 & 57 & 5.3 \\
\hline $2005-07-23$ & $22: 53: 35.1$ & 5.113 & 94.802 & 48 & 5.3 \\
\hline $2005-08-28$ & $04: 43: 41.0$ & 5.547 & 94.579 & 51 & 5.3 \\
\hline 2006-01-01 & $08: 47: 13.4$ & 4.735 & 95.144 & 51 & 5.5 \\
\hline $2006-05-13$ & 03:11:41.6 & 5.521 & 94.432 & 34 & 5.7 \\
\hline 2006-11-21 & $08: 32: 25.7$ & -6.216 & 154.748 & 31 & 5.6 \\
\hline 2007-09-15 & $14: 45: 29.2$ & -2.788 & 101.190 & 35 & 5.5 \\
\hline $2007-11-22$ & $23: 02: 13.0$ & 4.743 & 95.060 & 49 & 5.8 \\
\hline 2008-01-05 & 20:01:55.1 & 5.477 & 94.685 & 57 & 5.3 \\
\hline $2008-02-21$ & $23: 55: 36.5$ & -2.323 & 99.880 & 24 & 5.7 \\
\hline $2008-05-03$ & $03: 53: 34.9$ & -3.015 & 101.319 & 50 & 5.3 \\
\hline $2008-05-13$ & $10: 29: 19.3$ & 4.676 & 95.089 & 35 & 5.4 \\
\hline 2009-07-31 & $14: 56: 16.5$ & -8.795 & 108.744 & 17 & 5.4 \\
\hline 2010-08-11 & $19: 10: 21.4$ & -7.495 & 107.058 & 37 & 5.3 \\
\hline $2010-10-26$ & $23: 45: 38.5$ & -2.360 & 99.853 & 25 & 5.4 \\
\hline 2011-06-19 & $04: 15: 59.0$ & -3.247 & 101.361 & 57 & 5.4 \\
\hline 2011-08-06 & $02: 45: 55.6$ & -2.909 & 101.092 & 44 & 5.3 \\
\hline 2012-06-05 & 11:01:20.0 & 5.482 & 94.672 & 54 & 5.3 \\
\hline 2013-01-10 & $13: 47: 04.0$ & 4.712 & 95.115 & 38 & 5.7 \\
\hline 2013-02-08 & $17: 59: 51.7$ & -6.282 & 154.784 & 74 & 5.8 \\
\hline 2013-02-13 & $11: 00: 24.8$ & -2.990 & 101.297 & 55 & 5.3 \\
\hline $2013-10-22$ & $21: 15: 51.2$ & -6.345 & 154.966 & 50 & 5.5 \\
\hline $2013-12-29$ & $12: 49: 16.7$ & -5.605 & 153.986 & 24 & 5.5 \\
\hline
\end{tabular}

The original occurrence time and location information of the events are from the PDE catalog 
Table 2 Waveform doublets in Western Pacific area

\begin{tabular}{|c|c|c|c|c|c|c|c|}
\hline Doub_no. & $\mathrm{dT}$ & Doub_ID & cc_mantle(ave) & ddt_mantle & ddt_std & no_ddt & mantle_phases \\
\hline 1 & 0.12 & 200502_200412 & 0.98 & 0.002 & 0.019 & 10 & P,PP,S,PcP,ScP \\
\hline 2 & 0.37 & 200505_200501 & 0.96 & -0.021 & 0.015 & 10 & P,PP,S,PcP,ScP \\
\hline 3 & 0.44 & 200507_200502 & 0.98 & -0.018 & 0.034 & 10 & P,PP,S,PcP,ScP \\
\hline 4 & 0.46 & 200508_200503 & 0.97 & -0.015 & 0.013 & 10 & P,PP,S,PcP,ScP \\
\hline 5 & 0.56 & 200507_200412 & 0.97 & -0.007 & 0.030 & 10 & P,PP,S,PcP,ScP \\
\hline 6 & 1.37 & 200605_200412 & 0.98 & -0.019 & 0.025 & 10 & P,PP,S,PcP,ScP \\
\hline 7 & 2.36 & 200805_200601 & 0.99 & -0.010 & 0.013 & 10 & P,PP,S,PcP,ScP \\
\hline 8 & 2.55 & 200801_200506 & 0.94 & 0.027 & 0.029 & 5 & P,S,PcP,ScP \\
\hline 9 & 2.68 & 201010_200802 & 0.94 & -0.006 & 0.028 & 10 & P,PP,S,PcP,ScP \\
\hline 10 & 3.00 & 200801_200501 & 0.96 & -0.004 & 0.034 & 10 & P,PP,S,PcP,ScP \\
\hline 11 & 3.89 & 201108_200709 & 0.99 & -0.008 & 0.011 & 6 & P,PP,PcP,ScP \\
\hline 12 & 4.42 & 201206_200801 & 1.00 & 0.004 & 0.000 & 1 & PP,PcP \\
\hline 13 & 4.78 & 201302_200805 & 0.95 & -0.010 & 0.006 & 3 & P,PP,PcP \\
\hline 14 & 5.13 & 201301_200711 & 0.98 & -0.010 & 0.014 & 3 & PP,PcP,ScP \\
\hline 15 & 6.22 & 201302_200611 & 0.97 & -0.010 & 0.012 & 3 & P,PcP,PP \\
\hline 16 & 7.42 & 201206_200501 & 1.00 & -0.012 & 0.000 & 1 & PP,PcP \\
\hline 17 & 8.56 & 200907_200101 & 0.96 & 0.002 & 0.010 & 3 & $\mathrm{P}, \mathrm{PP}, \mathrm{PcP}$ \\
\hline 18 & 11.98 & 201008_199808 & 0.99 & 0.001 & 0.027 & 3 & pPKPdf,PKS,SKP \\
\hline 19 & 16.87 & 201106_199408 & 0.91 & 0.007 & 0.010 & 3 & $\mathrm{P}, \mathrm{PP}, \mathrm{ScP}$ \\
\hline 20 & 16.93 & 201312_199701 & 0.97 & 0.012 & 0.015 & 3 & P,PcP,PP \\
\hline 21 & 20.02 & 201310_199310 & 0.93 & -0.005 & 0.043 & 3 & $\mathrm{P}, \mathrm{PcP}, \mathrm{PP}$ \\
\hline
\end{tabular}

cc_mantle(ave) average cross-correlation value of all mantle phases, ddt_mantle average ddt value between mantle phases, ddt_std standard deviation of all the ddt_mantle values, no_ddt number of ddt values of mantle phases, mantle_phases mantle phases used in each doublet. The length of the time window in calculating the time shift and cross-correlation coefficients is $5 \mathrm{~s}$ roughly from the onset of the phase

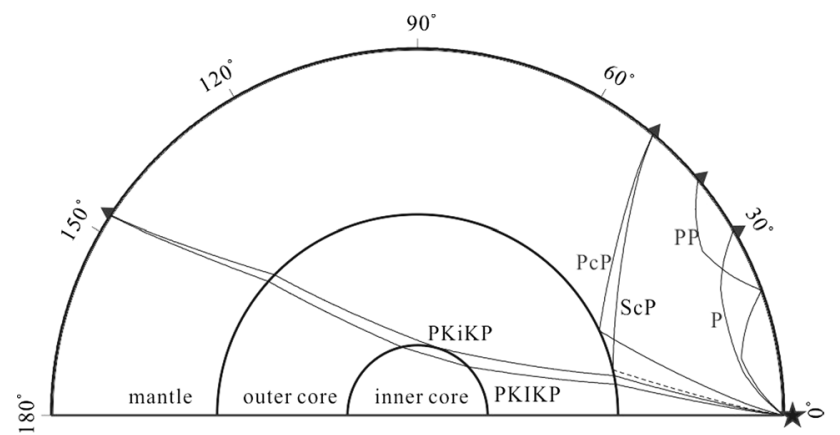

Fig. 1 An illustration of ray paths of mantle phases (P, ScP, PP, PcP) and core phases (PKIKP, PKiKP) used in this study. The source (star) and stations (triangles) are shown. The solid lines and dashed line represent the wave propagating as the $\mathrm{P}$ wave and the $\mathrm{S}$ wave, respectively

searching, the $\mathrm{CC}$ calculation was performed only for events pairs whose distance is less than $0.5^{\circ}$ using the PDE locations. Finally, we obtained 21 excellent doublets from 35 events (Table 1) with CC coefficients larger than 0.96 in the P-wave window (Table 2). The magnitude of the events ranges from 5.3 to 5.8. And the time separation between two events of a doublet spans from 0.12 to 20.02 years. We used other mantle phases including S, PP, PcP, ScP (Fig. 1) to check the robustness of these doublet pairs (see below).

\subsection{Relative time shift (ddt) measurements}

To detect the topography of the ICB, waveforms comprising the inner-core refracted phase PKIKP (or PKPdf) and inner-core reflected phase PKiKP (or PKPcd) (Fig. 1) are used. Since the ray paths of this phase pair are close in shallow, seismic heterogeneities in the mantle would affect the PKIKP and PKiKP phases in a similar way. The differential travel times of the PKiKP-PKIKP thus are most sensitive to seismic structure and topography at the ICB. We checked all the available stations globally within the distance range of $128^{\circ}-142^{\circ}$ (Fig. 2), where the two phases are best observed (Song and Helmberger 1992).

We followed the strategy outlined in Dai and Song (2008) and Song and Dai (2008) to formulate the relative time shift (ddt). We used the CC method to measure relative times of the same phase between the two events of the doublet. We first interpolated the seismograms to a new 


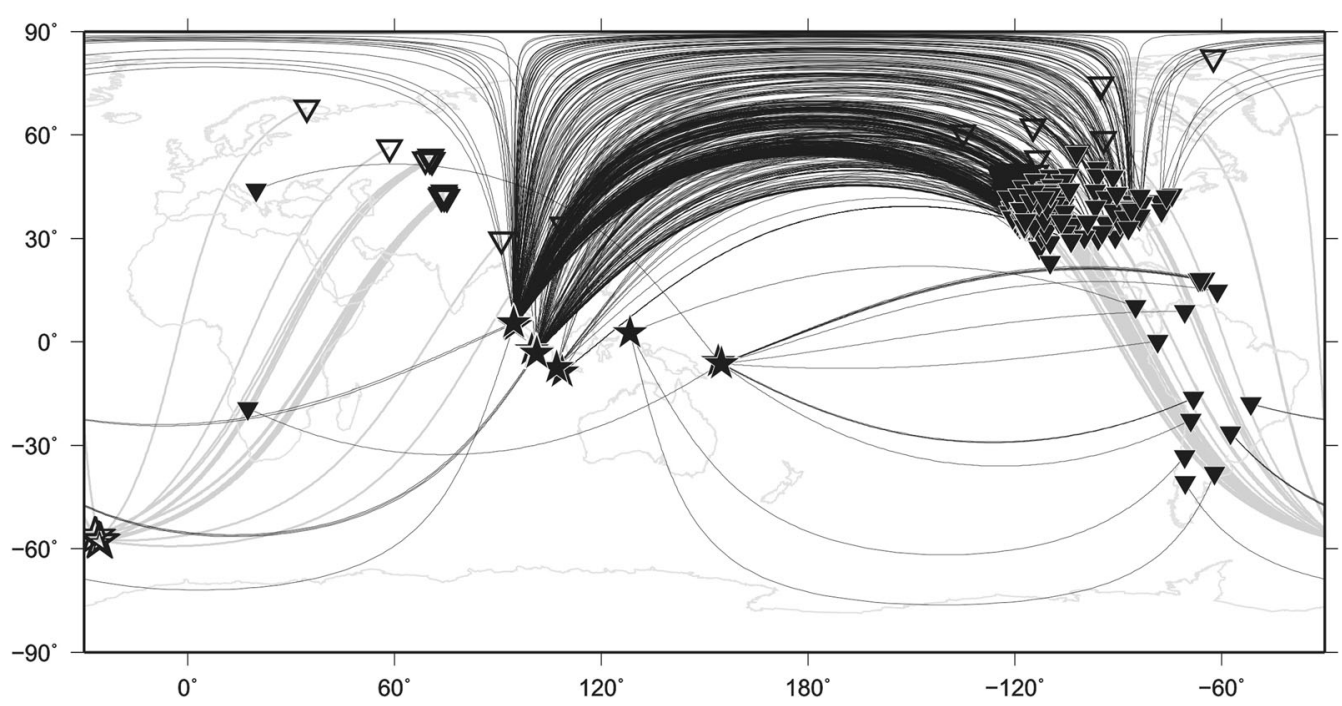

Fig. 2 Distribution of doublet events (stars), stations (inverted triangles), and ray paths (lines) used in this study. The doublets from the Western Pacific (WP) found in this study and South Sandwich Islands (SSI) from Song and Dai (2008) are shown in solid and open symbols, respectively

sampling interval of $0.0005 \mathrm{~s}$ before calculation to enhance the measurement precision. The length of time window ranges from 1 to $1.5 \mathrm{~s}$ for PKPdf phase and from 1.5 to $2.5 \mathrm{~s}$ for PKPcd phase depending on the distance and separation of the two phases. The relative time shift (ddt) between PKiKP and PKIKP phases from the two events of the doublet is defined as

$$
\begin{aligned}
d d t(c d-d f)= & \left(P K P c d_{2}-P K P c d_{1}\right)-\left(P K P d f_{2}\right. \\
& \left.-P K P d f_{1}\right) .
\end{aligned}
$$

The subscripts 1 and 2 represent the arrival times of the earlier and later events, respectively, for the same phase. A positive ddt value implies that the PKPcd phase of the later event arrives later than the earlier one relative to the PKPdf phase. The relative time shift can be applied to any two phases, such as mantle phases below, recorded at the same station for any doublet.

\section{Results and discussion}

3.1 Verification of doublet quality with ddt of mantle phases

To verify the quality of our new doublets in the WP, we checked the similarity of as many mantle phases as possible, which included $\mathrm{P}, \mathrm{S}, \mathrm{PcP}, \mathrm{PP}, \mathrm{ScP}$. The additional mantle phases (besides $\mathrm{P}$ ) at the same station are particularly useful, as they can be used to examine relative time shifts (ddts) that are not influenced by the inner core. Thus, we checked systematically whether there were available mantle phases for these doublets at station CHTO. In the end, we used only the doublets that have two or more mantle phases with sufficient quality recorded at station CHTO.

Table 2 summarizes all of our average measurements of the relative time shifts and CC coefficients for mantle phases. The time shift for one phase between two events of the doublet is relative to the origin times of the two events as listed in Table 1. The CC values of core phases (PKPdf, PKPcd) and mantle phases (P, PcP, PP, ScP) are generally over 0.90 , indicating high similarity between the two events of the every doublet that we found in the WP.

Figure 3 shows typical example waveforms of mantle phases P, PcP, PP, ScP phases from three doublets: 201108_200709 (\#11, Table 2)(a), 200907_200101 (\#17) (b) and 201312_199701 (\#20) (c). The time lapses of these doublets range from 4 to 17 years. As we can see, all of these mantle phases are very consistent over a long time window for these doublets. Note the quality of the doublets is about average or above with the CC coefficients of 0.99 , $0.96,0.97$, respectively. Note also the 201310_199310 pair 

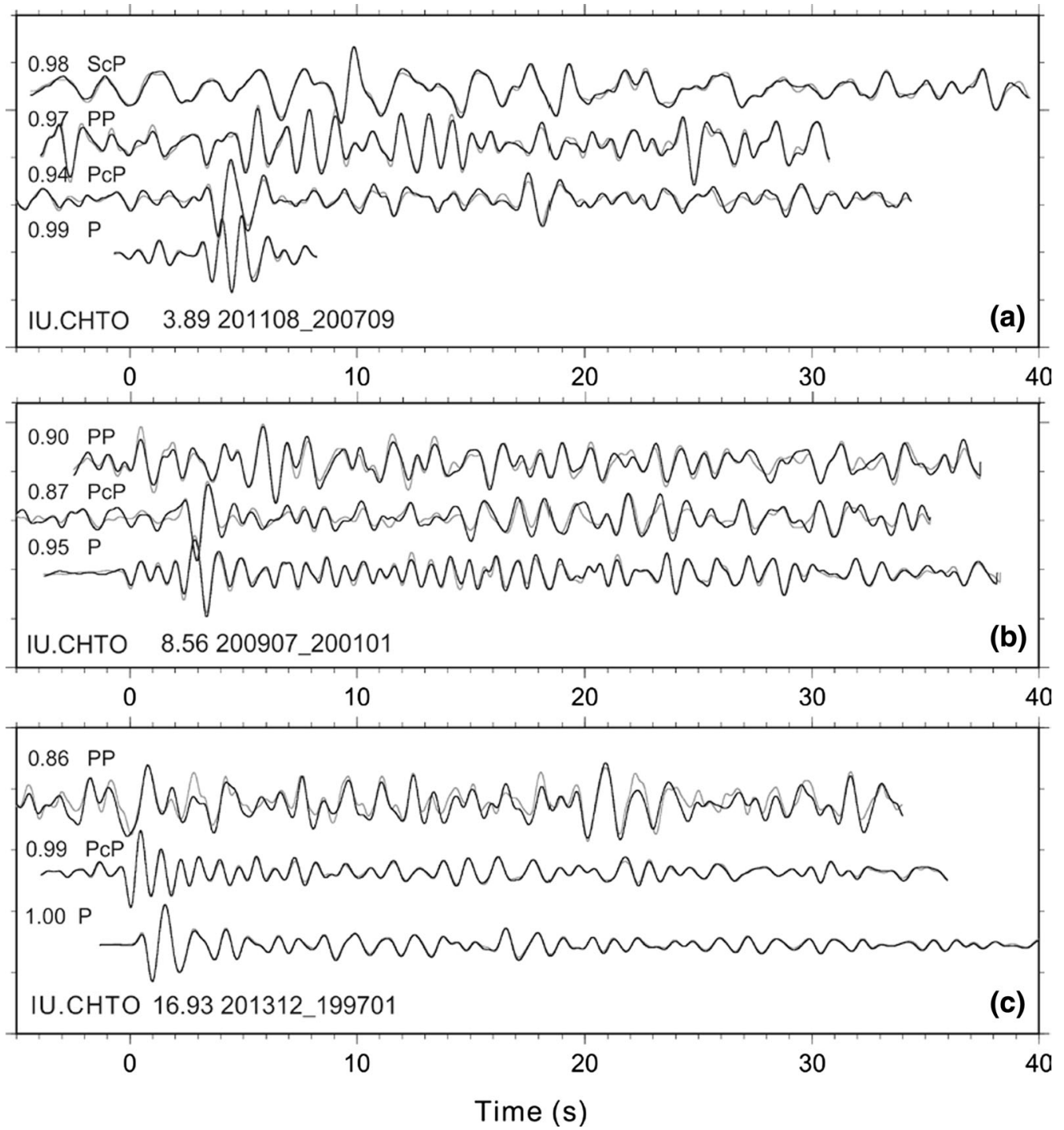

Fig. 3 Examples of mantle phases (P, PP, PcP, ScP) for a doublet 201108_200709, b doublet 200907_200101, and c doublet 201312_199701 (labeled) at station CHTO. The waveforms from the earlier (gray) and later (black) events are overlaid. The CC coefficients (numbers above the traces) are calculated using the whole waveform segments appeared in the time window

has a large difference in focal depths from the PDE catalog (50 and $102 \mathrm{~km}$, respectively). We have checked carefully the later-arriving wave packets after $\mathrm{P}$ waves, which show good matches between the two events, suggesting that the focal depths must be similar.

\subsection{Temporal shifts of PKPcd and PKPdf waves}

Using the method described above, we obtained a total of 614 new ddt measurements from the WP doublets
(Table 3). The relative time shifts between PKPcd and PKPdf phase show different features for doublets from the WP and the SSI. In general, the ddt values for the paths from earthquakes in WP are very small (all within $\pm 0.025 \mathrm{~s}$ ) regardless of the length of year lapse between two doublet events (Fig. 4). And the scatter of the ddt values is similar for all doublets of these paths. In contrast, the distribution of ddt values for the doublets from the SSI presents a different view. The ddt values appear more scattered and much larger (up to $-0.12 \mathrm{~s}$ ) when the year lapse is more than 6 years. 
Table 3 Detailed information of waveform doublets in Western Pacific area

\begin{tabular}{|c|c|c|c|c|c|c|c|c|c|}
\hline Doub_no. & $\mathrm{dT}$ & Doub_ID & No._stat & df_dt & df_cc & cd_dt & cd_cc & ddt(cd-df) & ddt(cd-df)_std \\
\hline 1 & 0.12 & 200502_200412 & 31 & 0.056 & 0.98 & 0.056 & 0.98 & 0.000 & 0.011 \\
\hline 2 & 0.37 & 200505_200501 & 56 & -1.002 & 0.98 & -1.004 & 0.98 & -0.002 & 0.012 \\
\hline 3 & 0.44 & 200507_200502 & 19 & -0.782 & 0.98 & -0.779 & 0.98 & 0.002 & 0.012 \\
\hline 4 & 0.46 & 200508_200503 & 11 & 0.576 & 0.95 & 0.570 & 0.96 & -0.006 & 0.015 \\
\hline 5 & 0.56 & 200507_200412 & 23 & 0.003 & 0.98 & 0.003 & 0.98 & 0.001 & 0.013 \\
\hline 6 & 1.37 & 200605_200412 & 84 & -0.453 & 0.99 & -0.451 & 0.99 & 0.001 & 0.009 \\
\hline 7 & 2.36 & 200805_200601 & 19 & 1.763 & 0.98 & 1.760 & 0.98 & -0.003 & 0.008 \\
\hline 8 & 2.55 & 200801_200506 & 8 & 0.399 & 0.97 & 0.410 & 0.98 & 0.011 & 0.008 \\
\hline 9 & 2.68 & 201010_200802 & 6 & -0.552 & 0.97 & -0.552 & 0.98 & -0.000 & 0.010 \\
\hline 10 & 3.00 & 200801_200501 & 11 & -3.090 & 0.95 & -3.095 & 0.98 & -0.004 & 0.014 \\
\hline 11 & 3.89 & 201108_200709 & 39 & -0.658 & 0.98 & -0.658 & 0.97 & -0.000 & 0.010 \\
\hline 12 & 4.42 & 201206_200801 & 25 & 0.052 & 0.99 & 0.052 & 0.99 & 0.000 & 0.012 \\
\hline 13 & 4.78 & 201302_200805 & 54 & -0.685 & 0.97 & -0.686 & 0.97 & -0.001 & 0.011 \\
\hline 14 & 5.13 & 201301_200711 & 59 & 1.381 & 0.98 & 1.380 & 0.97 & -0.002 & 0.010 \\
\hline 15 & 6.22 & 201302_200611 & 9 & -5.303 & 0.99 & -5.302 & 0.99 & 0.001 & 0.011 \\
\hline 16 & 7.42 & 201206_200501 & 19 & -2.941 & 0.97 & -2.939 & 0.99 & 0.002 & 0.009 \\
\hline 17 & 8.56 & 200907_200101 & 1 & 1.523 & 0.97 & 1.503 & 0.97 & -0.020 & 0.000 \\
\hline 18 & 11.98 & 201008_199808 & 8 & 2.937 & 0.97 & 2.938 & 0.97 & 0.000 & 0.014 \\
\hline 19 & 16.87 & 201106_199408 & 2 & 0.090 & 0.97 & 0.108 & 0.92 & 0.018 & 0.001 \\
\hline 20 & 16.93 & 201312_199701 & 1 & 3.482 & 0.96 & 3.472 & 0.99 & -0.010 & 0.000 \\
\hline 21 & 20.02 & 201310_199310 & 2 & 4.373 & 0.99 & 4.377 & 0.95 & 0.004 & 0.005 \\
\hline
\end{tabular}

$\mathrm{dT}$ refers to the occurrence time gap between the two doublet events. Doublet_ID is named with the occurrence year and month of the two doublet events. No._stat implies the total number of receiver stations to calculate df_cc, df_dt, cd_cc, cd_dt, which represent the averages of PKPdf's cc (df_cc), PKPdf's time shift (df_dt), PKPcd's cc (cd_cc), PKPcd's time shift (cd_dt) of each two doublet events, respectively; ddt (cd--df) refers to the average time shift between PKPcd and PKPdf phase; ddt (cd--df)_std is the standard deviation of ddt (cd--df)

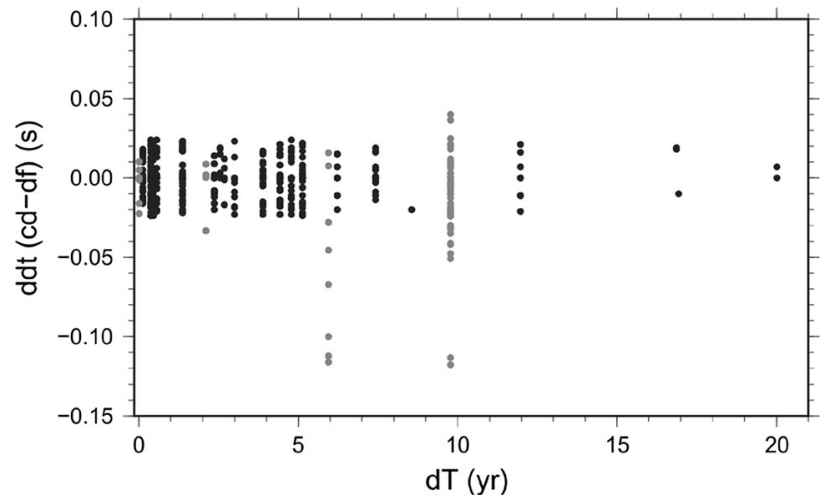

Fig. 4 Relative time shift (ddt) between PKPcd and PKPdf phases as a function of time lapse between the two events of the doublet. The doublets are from the WP (black dots) and from the SSI (gray dots) published in Song and Dai (2008). The four doublets from the SSI are d-0-2000, t1-2-9799, t2-6-9804, d-10-9303
The difference between the paths from WP and SSI earthquakes is obvious in the waveforms (Figs. 5, 6). Figure 5 compares PKPdf and PKPcd waveforms for doublet 201008_199808 in WP with a large time lapse (about 12 years) and those from a SSI doublet (t2-6-9804) with a moderate time lapse (about 6 years) from Song and Dai (2008). All the waveforms of the WP doublet are very similar. However, as discussed in Song and Dai (2008), the waveforms of the doublet t2-6-9804 are variable, which are similar at station WHY but show big difference at stations UCH and AML. Figure 6 plots PKPdf and PKPcd waveforms of doublets with different time lapses at a few selected stations. Regardless the time lapses (greater than 6 years for three pairs), the waveform of the pairs are very similar with only small temporal shifts. 

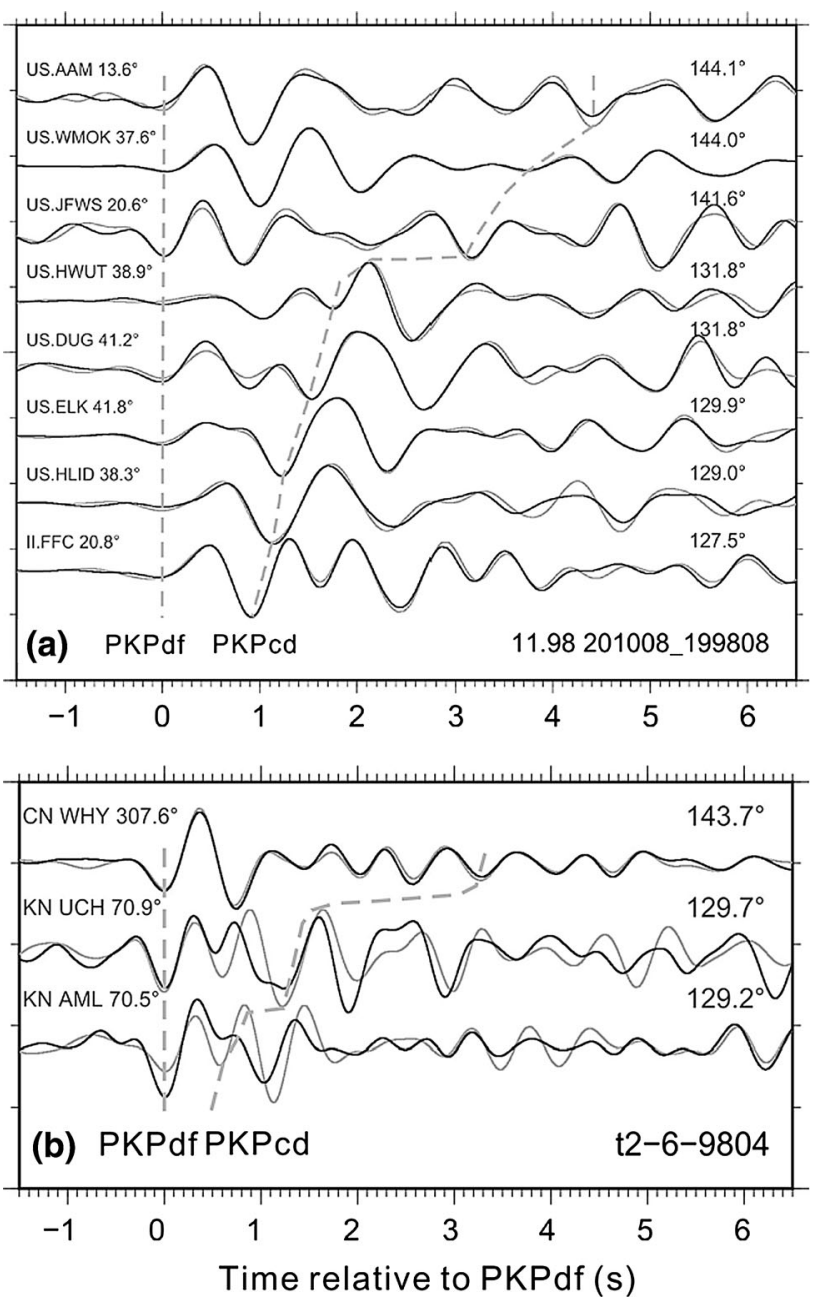

Fig. 5 Example waveforms of PKPdf and PKPcd for doublets with relatively large lapse times. The doublets are a double 201008_199808 from Western Pacific and b t2-6-9804 from the SSI (Song and Dai 2008). The waveforms from the earlier (gray) and later (black) events of each doublet are shown. The net code, station code, azimuth, and distance are labeled for each station. The traces are aligned with PKPdf phase and sorted according to distance

To examine the global distribution of the temporal shifts, we plotted the ddt values from our new measurements and Song and Dai (2008) at the turning points of the ray paths at the ICB (Fig. 7). All the ddt values are small when the year lapse between two events of each doublet is less than 3 years. When year lapse is greater than 6 years, the temporal change is variable among different regions of the ICB. A few places show large temporal shifts, i.e., beneath African and Central America area regions. Furthermore, the ddt values show rapid variation within the two regions. The temporal shifts in all other sampling regions are small.

The spatial variability of the temporal shifts can also be observed from the CC coefficients (Fig. 8). The CC values are all high when the year lapse is smaller than 3 years. The values are also high for large time lapses except some central America and Africa samples.

\section{Conclusion and discussion}

We examined a total of 21 new high-quality doublets in the WP recorded at stations within the distance range of $128^{\circ}$ $142^{\circ}$. Combined with measurements by Song and Dai (2008) for doublets occurred in the SSI using the doublet method, our basic observations can be summarized as follows. When the year lapse between two doublet events is less than 3 years, all the sampled ICB regions show little temporal change. When the year lapse is larger than 6 years, large temporal changes can be observed in the ICB regions under Africa and Central America. Meanwhile, the temporal change remains subtle for the ICB regions beneath Atlantic Ocean, Indian Ocean, and the northeast of Eurasia. Thus, the topography of the ICB appears rather localized in certain regions.

These observations can be explained by the following two possible mechanisms proposed previously (Song and Dai, 2008). (1) Significant topography of the ICB in certain regions. Most regions may have relatively uniform structure, like the ICB regions sampled by the WP doublets. That is why the temporal change for these samples remains subtle even when the year lapse is over 6 years. On the contrary, significant ICB topography exists in some other regions, such as Africa and Central America. The rotation of the inner core shifts the topography when the year lapse is long enough, causing observable tem- 

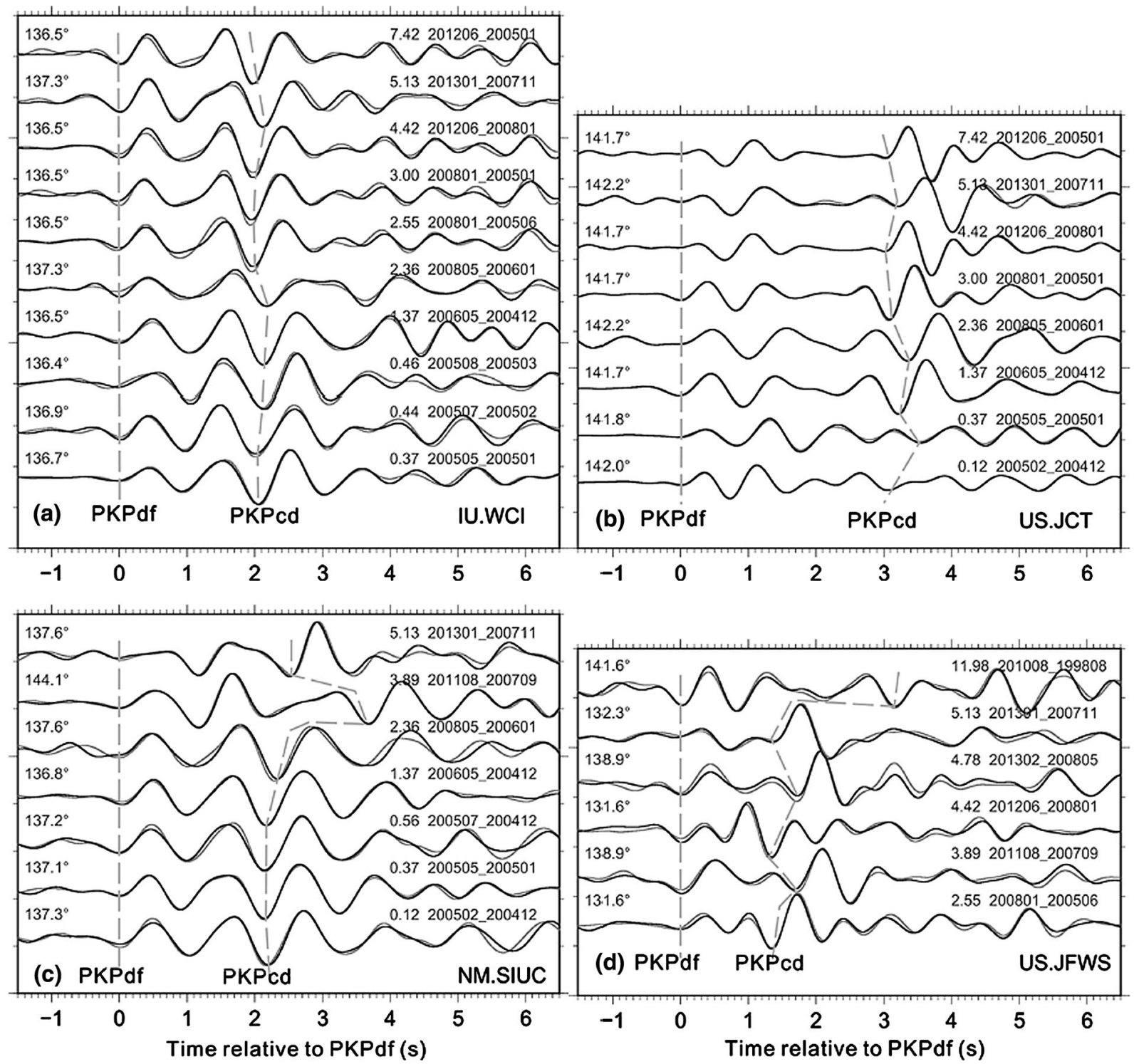

Fig. 6 Examples of doublet waveforms at five stations (WCI, JCT, SIUC, JFWS, LRAL). The waveforms from the earlier (gray) and later (black) events of each doublet are shown. The distance, year lapse, and doublet_ID are labeled for each station. The traces are aligned with PKPdf phase and sorted according to the year lapse between the event pair

poral changes. (2) The ICB has a transient slurry structure (Loper and Roberts 1981). That is in some regions, the ICB topography is a mixture of molten materials and solidified patches of iron crystals. The turbulence of the outer core convection may shift the newly solidified materials at the base of the outer core, leading to the variation in temporal changes of the ICB when the year lapse is relatively large. More samples of the ICB will be critical in future research in understanding this dynamic region. 

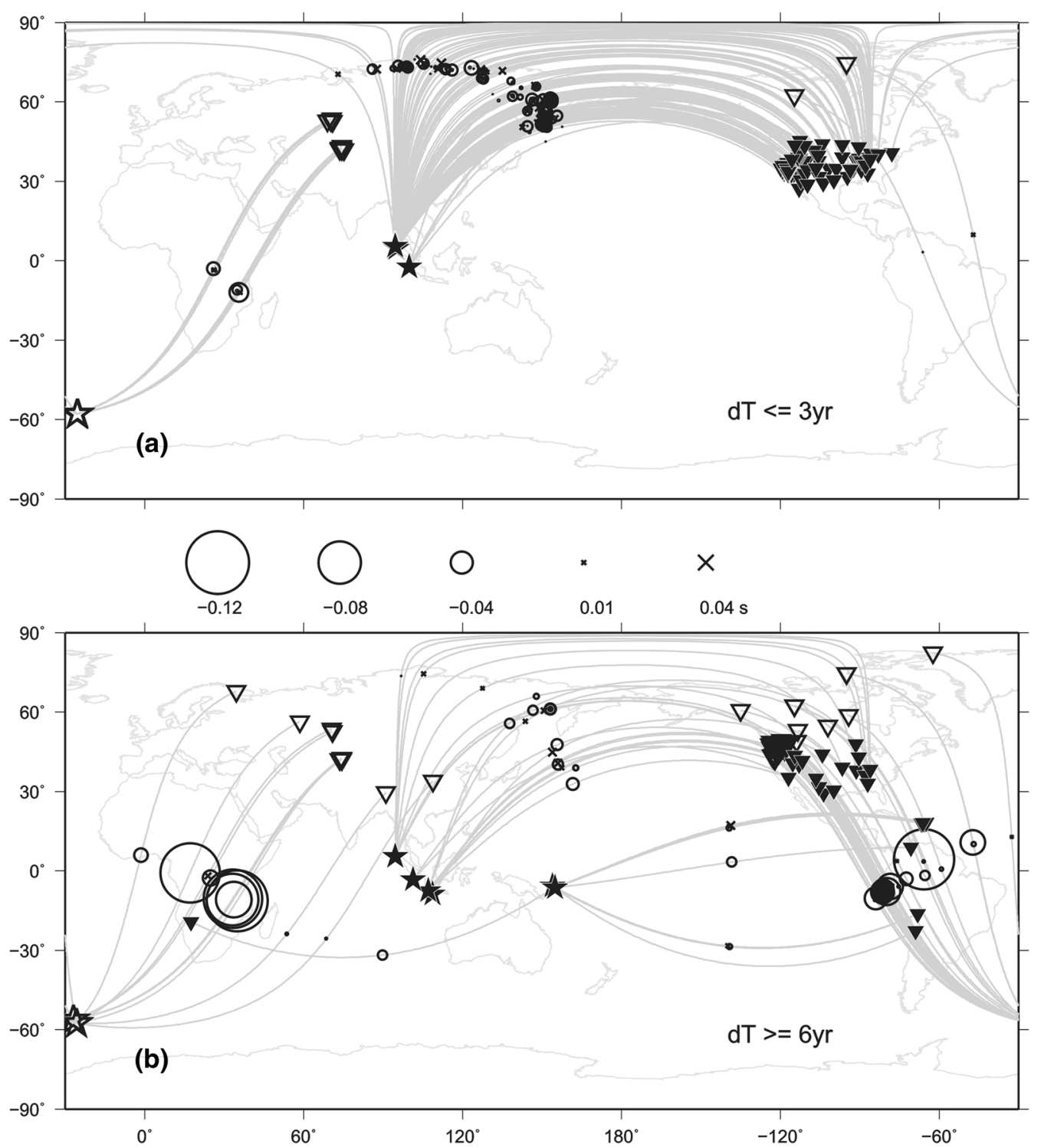

Fig. 7 Maps of relative time shifts (ddt) between PKPcd phase and PKPdf phase for doublets with a relatively short time separations (year lapse $\leq 3$ years) and $\mathbf{b}$ relatively long time separations (year lapse $\geq 6$ years). Shown are earthquakes (stars), stations (inverted triangles), ray paths (gray lines), and ddt values (circles: negative or crosses: positive). The symbols related to the WP and the SSI (Song and Dai 2008) earthquakes and stations are solid and open, respectively. The ddt values (circles and crosses) are plotted at the ICB bounce points of the ray paths and the symbol size is proportional to the relative time shift 

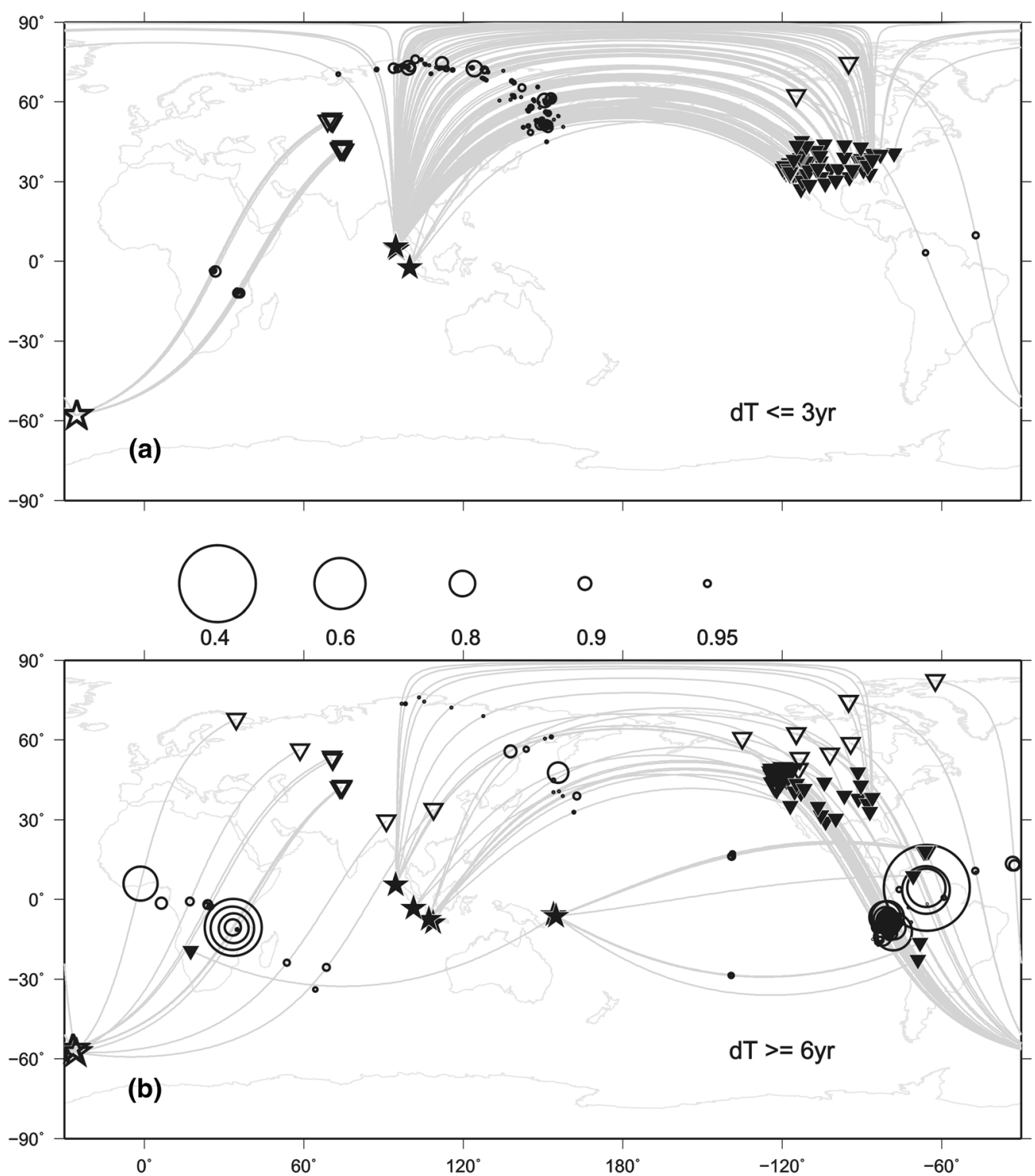

Fig. 8 CC coefficients of PKPcd phases for doublets with a relatively short time separation (year lapse $\leq 3$ years) and $\mathbf{b}$ relatively long time separation (year lapse $\geq 6$ years). The earthquakes (stars), stations (inverted triangles), ray paths (gray lines), and CC values (circles) are shown. The symbols related to the WP and the SSI (Song and Dai 2008) are solid and open, respectively. The CC values (circles) are plotted at the ICB bounce points of the ray paths and the symbol size is proportional to (1-CC) value

Acknowledgments All the data used in this study were obtained from Incorporated Research Institutions for Seismology Data Management Center (http://iris.edu). The doublet search program was based on a computer program by Shao Lin from Nanjing University. This research was supported by the Natural Science Foundation of China (41330209), the Ministry of Science and Technology of China (2014CB845901), and the China Earthquake Administration.

Open Access This article is distributed under the terms of the Creative Commons Attribution 4.0 International License (http://creativecommons.org/licenses/by/4.0/), which permits unrestricted use, distribution, and reproduction in any medium, provided you give appropriate credit to the original author(s) and the source, provide a link to the Creative Commons license, and indicate if changes were made.

\section{References}

Cao A, Masson Y, Romanowicz B (2007) Short wavelength topography on the inner-core boundary. Proc Natl Acad Sci 104(1):31-35

Cormier VF, Xu L, Choy GL (1998) Seismic attenuation of the inner core: viscoelastic or stratigraphic? Geophys Res Lett 25(21):4019-4022

Dai W, Song X (2008) Detection of motion and heterogeneity in Earth's liquid outer core. Geophys Res Lett 35(16):L16311

Dai Z, Wang W, Wen L (2012) Irregular topography at the Earth's inner core boundary. Proc Natl Acad Sci 109(20):7654-7658

Dziewonski AM, Anderson DL (1981) Preliminary reference Earth model. Phys Earth Planet Inter 25(4):297-356 
Fearn DR, Loper DE, Roberts PH (1981) Structure of the Earth's inner core. Nature 292:232-233

Glatzmaier GA, Roberts PH (1995) A three-dimensional convective dynamo solution with rotating and finitely conducting inner core and mantle. Phys Earth Planet Inter 91(1):63-75

Irving JCE, Deuss A (2011) Hemispherical structure in inner core velocity anisotropy. J Geophys Res 116(B4):B04307

Jacobs JA (1953) The Earth's inner core. Nature 172(4372):297-298

Jiang G, Zhao D (2012) Observation of high-frequency PKiKP in Japan: insight into fine structure of inner core boundary. J Asian Earth Sci 59:167-184

Koper KD, Franks JM, Dombrovskaya M (2004) Evidence for smallscale heterogeneity in Earth's inner core from a global study of PKiKP coda waves. Earth and Planetary Science Letters 228(3-4):227-241

Krasnoshchekov DN, Kaazik PB, Ovtchinnikov VM (2005) Seismological evidence for mosaic structure of the surface of the Earth's inner core. Nature 435(7041):483-487

Loper DE, Roberts PH (1981) A study of conditions at the inner core boundary of the Earth. Phys Earth Planet Inter 24(4):302-307

Niu F, Wen L (2001) Hemispherical variations in seismic velocity at the top of the Earth's inner core. Nature 410(6832):1081-1084

Poupinet G, Ellsworth WL, Frechet J (1984) Monitoring velocity variations in the crust using earthquake doublets: an application to the Calaveras Fault, California. J Geophys Res (1978-2012) 89(B7):5719-5731

Song X, Dai W (2008) Topography of Earth's inner core boundary from high-quality waveform doublets. Geophys J Int 175(1):386-399
Song X, Helmberger DV (1992) Velocity structure near the inner core boundary from waveform modeling. Journal of Geophysical Research-Solid Earth 97(B5):6573-6586

Song X, Helmberger DV (1995) Depth dependence of anisotropy of Earth's inner core. J Geophys Res 100(B6):9805-9816

Song X, Richards PG (1996) Seismological evidence for differential rotation of the Earth's inner core. Nature 382(6588):221-224

Souriau A, Poupinet G (1991) The velocity profile at the base of the liquid core from PKP (BC + Cdiff) data: an argument in favour of radial inhomogeneity. Geophys Res Lett 18(11):2023-2026

Stevenson DJ (1987) Limits on lateral density and velocity variations in the Earth's outer core. Geophys J Int 88(1):311-319

Stevenson DJ, Spohn T, Schubert G (1983) Magnetism and thermal evolution of the terrestrial planets. Icarus 54(3):466-489

Vidale JE, Earle PS (2005) Evidence for inner-core rotation from possible changes with time in PKP coda. Geophys Res Lett 32(1):L01309

Vidale JE, Dodge DA, Earle PS (2000) Slow differential rotation of the Earth's inner core indicated by temporal changes in scattering. Nature 405(6785):445-448

Wen L (2006) Localized temporal change of the Earth's inner core boundary. Science 314(5801):967-970

Yoshida S, Sumita I, Kumazawa M (1996) Growth model of the inner core coupled with the outer core dynamics and the resulting elastic anisotropy. J Geophys Res 101(B12):28085-28103

Zhang J, Song X, Li Y et al (2005) Inner core differential motion confirmed by earthquake waveform doublets. Science 309(5739):1357-1360 